\section{FASES PRÉ-ANALITICA, ANALITICA E PÓS-ANALÍTICA NO MONITORAMENTO LABORATORIAL DA ANTICOAGULAÇÃO COM ANTAGONISTAS DA VITAMINA K}

\author{
PRE-ANalytical, anALYTICAL, AND POST-ANaLYTICAL PHASES \\ IN LABORATORY MONITORING OF ANTICOAGULANT THERAPY WITH \\ VITAMIN K ANTAGONISTS
}

Jonas Michel Wolf1', Lucas Michel Wolf²

\section{RESUMO}

A trombose é uma doença caracterizada por eventos de hipercoagulabilidade. A terapêutica anticoagulante oral com antagonistas da vitamina K (AVKs) é amplamente indicada para prevenção e/ou controle de distúrbios da coagulação. O manuseio de administração dos AVKs é difícil devido à complexidade da definição da dose. Em geral, o monitoramento de indivíduos submetidos à terapêutica com AVK é realizado pela determinação do tempo de protrombina, em que se avalia o grau de anticoagulação através do coeficiente internacional normatizado. Invariavelmente, o fluxo do processamento laboratorial, que compreende as fases pré-analítica, analítica e pós-analítica, é importante para a fidedignidade dos resultados, repercutindo na conduta médica de forma determinante. O objetivo deste estudo foi a realização de uma revisão da literatura científica descritiva utilizando bases de dados eletrônicos para busca de materiais científicos, como Google Scholar, MEDLINE, LILACS, PubMed, SciELO e Science Direct. Abordamos alguns aspectos relacionados ao fluxo analítico da monitoração laboratorial em um laboratório de análises clínicas. Em conclusão, é necessária uma apropriada condução das fases analíticas para que seja possível o sucesso terapêutico com uso dos AVKs.

Palavras-chave: Tempo de protrombina; coeficiente internacional normatizado; fases analíticas

\begin{abstract}
Thrombosis is a disease characterized by hypercoagulable events. Oral anticoagulant therapy with vitamin $\mathrm{K}$ antagonists (VKAs) is widely indicated for prevention and/or control of coagulation disorders. The administration of VKAs is difficult because of the complexity of dose setting. In general, individuals submitted to VKA therapy are monitored by prothrombin time, in which the degree of anticoagulation is assessed by the international normalized ratio. Invariably, the flow of laboratory processing, which comprises pre-analytical, analytical, and post-analytical phases, is of importance to the trustworthiness of results, with significant consequences to the medical practice. The purpose of this study was to carry out a review of the scientific literature using electronic databases to search for scientific materials, such as Google Scholar, MEDLINE, LILACS, PubMed, SciELO, and Science Direct. We discuss some aspects related to the analytical flow of laboratory monitoring in a clinical laboratory. In conclusion, appropriate management of the analytical phases is necessary so that therapeutic success using VKAs is possible.
\end{abstract}

Keywords: Prothrombin time; international normalized ratio; analytical phases
Clin Biomed Res. 2017;37(2):125-131

1 Programa de Pós-graduação em Biologia Celular e Molecular Aplicada à Saúde, Universidade Luterana do Brasil (ULBRA). Canoas, RS, Brasil.

2 Laboratório de diagnóstico molecular, Universidade Luterana do Brasil (ULBRA). Canoas, RS, Brasil.

Autor correspondente: Jonas Michel Wolf jonasmwolf@gmail.com Programa de Pós-graduação em Biologia Celular e Molecular Aplicada à Saúde, Universidade Luterana do Brasil (ULBRA)

Av. Farroupilha, 8001

92425-900, Canoas, RS, Brasil. 
Os antagonistas da vitamina K (AVKs) são fármacos anticoagulantes orais utilizados em uma amplitude de condições frequentes no manejo clínico de eventos trombóticos ${ }^{1}$. As indicações atualmente direcionadas para a anticoagulação incluem a prevenção e o tratamento de tromboembolismo venoso, trombose venosa profunda e embolia pulmonar, a profilaxia de fenômenos cardioembólicos nos pacientes com fibrilação atrial ou próteses valvulares mecânicas e a prevenção secundária nos doentes com síndromes coronárias agudas ou submetidos à intervenção coronária percutânea ${ }^{1-5}$. Além disso, os AVKs são indicados na prevenção primária de recorrência do infarto agudo do miocárdio ${ }^{6}$. Devido à detecção de situações clínicas que favorecem a gênese de eventos trombóticos, as indicações para uso crônico de AVK vêm se difundindo de forma abrangente, o que exige um controle adequado e seguro desse tipo de intervenção farmacológica ${ }^{6,7}$.

O monitoramento laboratorial, por intermédio da mensuração do tempo de protrombina (TP) e seus parâmetros, é um importante passo na terapia com AVK. No entanto, diversos fatores estão envolvidos na resposta farmacológica do paciente e, inexoravelmente, no controle da anticoagulação $0^{6-9}$. Nesse contexto, a integridade da perfeita efetividade do fluxo processual, no que diz respeito à confiabilidade e qualidade dos resultados, é dependente de possíveis erros nas fases pré-analítica, analítica e pós-analítica. A fase pré-analítica atua como protagonista nos índices de erros relatados nas etapas laboratoriais ${ }^{10}$. Os ensaios de coagulação são intensamente vulneráveis a erros pré-analíticos que determinam importantes influências nos resultados ${ }^{11-13}$. Contextualizando, Carraro e Plebani ${ }^{14}$ definiram que $12,3 \%$ dos erros analíticos podem influenciar significativamente a conduta médica e, com isso, ocasionar repercussões clínicas importantes no paciente. Podemos entender, portanto, a relevância de tal impacto nos exames de coagulação ${ }^{11-13}$. A partir desse panorama, o enfoque da presente pesquisa foi realizar um levantamento descritivo da literatura científica a respeito das possíveis variáveis pré-analíticas, analíticas e pós-analíticas no monitoramento laboratorial da terapêutica com AVK.

\section{MÉTODOS}

Este trabalho consiste em uma revisão da literatura científica descritiva, focada nos aspectos relacionados às fases analíticas da monitoração laboratorial com AVK. A seleção dos materiais científicos foi efetuada a partir de buscas em plataformas eletrônicas, tais como: Google Scholar, Medical Literature Analysis and Retrieval System Online (MEDLINE), Literatura Latino-Americana e do Caribe em Ciências da Saúde (LILACS), Scientific Eletronic Library Online (SciELO), US National Library of Medicine National Institutes of Health (NCBI/PubMed) e Science Direct. Publicações científicas que contemplassem o objetivo do presente estudo foram selecionadas. Foram utilizadas as seguintes palavras-chave: "oral anticoagulation", "laboratory monitoring of anticoagulation", "coagulation", "thrombosis", "phase analytical laboratory", "pre-analytical phase in the laboratory", "Prothrombin time", "International Sensitivity Index" e "International Normalized Ratio". Estudos ou relatos de casos, correspondências, comentários ou estudos não publicados não foram incluídos. O Quadro 1 informa com maiores detalhes como foi feita a seleção dos materiais científicos utilizados para a redação desta revisão, de acordo com as pesquisas nas plataformas eletrônicas. $O$ período de seleção compreendeu os últimos 20 anos.

\section{REVISÃO DA LITERATURA}

\section{Metodologia do Ensaio Tempo de Protrombina (TP)}

A metodologia analítica do TP baseia-se na mensuração do tempo que um plasma privado de cálcio, devido à descalcificação, demora até atingir a formação de um coágulo, incubado a $37^{\circ} \mathrm{C}$ e na presença de um aporte de tromboplastina tecidual e

Quadro 1: Lista quantitativa de materiais científicos selecionados e excluídos no presente estudo.

\begin{tabular}{|l|c|c|c|c|}
\hline \multirow{2}{*}{$\begin{array}{c}\text { Plataformas de } \\
\text { pesquisa }\end{array}$} & $\mathbf{n}$ & Selecionados & $\mathbf{n}$ & Excluídos* \\
\cline { 2 - 5 } & 30 & 58,8 & 20 & 58,9 \\
\hline NCBI/PubMed & 6 & 11,8 & 5 & 14,7 \\
\hline SciELO & 7 & 13,7 & 5 & 14,7 \\
\hline Google Scholar & 3 & 5,9 & 1 & 2,9 \\
\hline Science Direct & 3 & 5,9 & 2 & 5,9 \\
\hline MEDLINE & 2 & 3,9 & 1 & 2,9 \\
\hline LILACS & 51 & 100 & 34 & 100 \\
\hline Total & & n & & \\
\hline
\end{tabular}

*Estudos ou relatos de casos, correspondências, comentários ou estudos não publicados foram excluídos. 
cálcio. Por consequência, o fator de coagulação VII é ativado, originando uma dupla molecular (complexo FT-FVIla), que terá a função de ocasionar a ativação dos fatores IX e X. O fator $\mathrm{Xa}$, em conjunto com fosfolipídios do fator tecidual, fator Va e cálcio, contribui para a formação de um conjunto ativador de protrombina que a converte em trombina (fator Ila). A trombina, por sua vez, é essencial para a conversão bioquímica do fibrinogênio em fibrina e a estabilização do coágulo ${ }^{15}$.

A tromboplastina é um reagente originário do cérebro humano, de boi, de macaco ou de coelho. O fator tecidual está presente nesse reagente, sendo composto por uma fração proteica e outra de fosfolipídios que mimetizam in vivo a função do fator 3 plaquetário, no que concerne à ativação de fatores que se combinam a fosfolipídios de membrana ${ }^{15,16}$.

A expressão dos resultados do TP pode ser realizada das seguintes formas ${ }^{15,16}$ :

a) Tempo pelo método de Quick, em segundos;

b) Percentual de atividade em relação a um plasma normal (no qual foi considerada uma atividade de $100 \%$ ), sendo necessário tangenciar uma curva de atividade utilizando um pool de plasmas normais obtidos e titulados com salina fisiológica $(0,9 \%)$;

c) Coeficiente Internacional Normatizado (CIN).

Os valores de referência dos parâmetros desses ensaios estão apresentados a seguir ${ }^{17}$ :

a) Tempo de protrombina (Quick) - 10-14 segundos;

b) Atividade (\%) - 70-100\%;

c) CIN - 1,0-1,08 em pessoas hígidas e 2,0-3,5 em pacientes submetidos à terapêutica com AVK.

Os valores de referência devem ser delimitados por cada laboratório, e os resultados variantes são dependentes do tipo de equipamento utilizado. Os resultados deverão ser formalizados em CIN, proporcionando, assim, a normatização da variação entre diferentes laboratórios. A determinação dos valores de índice de sensibilidade internacional (ISI) é efetuada através de comparação com um material referencial de tromboplastina primária ${ }^{15,17}$.

A sensibilidade do reagente é inversamente proporcional ao valor de ISI. Na grande maioria, situase entre 1,0-2,0, sendo que quanto mais próximo de 1,0 , mais sensível é o reagente utilizado ${ }^{15,16}$.

\section{Fase Pré-analítica Aplicada à Determinação do TP}

A fase pré-analítica é a etapa que concentra o maior número de erros que podem afetar o diagnóstico ${ }^{15,18,19}$. A explicação consiste na inexistência de automação e na dificuldade de controle do processo, incluindo a variabilidade da condição de jejum do paciente ou a omissão do paciente a respeito de questionamentos que são essenciais para garantir a qualidade do exame ${ }^{18,20-23}$.

Causas de erros pré-analíticos, como tempo de armazenamento $(78,6 \%)$, tempo de torniquete $(78,6 \%)$, metodologia de flebotomia $(64,3 \%)$, falta de informação do paciente $(64,3 \%)$, incorreta relação sangue/anticoagulante $(57,0 \%)$ e tubos inadequados (50\%), são reportados por diferentes estudos ${ }^{24-27}$.

As variáveis pré-analíticas são determinantes nos resultados dos testes de coagulação, com destaque para a flebotomia, seguida do intervalo entre a coleta e a realização do ensaio laboratorial. Além disso, os fatores a seguir são importantes aspectos da efetividade dos resultados laboratoriais ${ }^{11-13}$ :

a) Anticoagulantes: o tubo com citrato de sódio é o de escolha. Habitualmente, há uma quantidade de citrato para um volume específico de sangue, que será completado em uma proporção de uma parte de citrato para nove de sangue. Alterações na proporção sangue-anticoagulante influenciam os resultados de forma importante. Para pacientes com eritrocitose, com hematócrito superior a 55,0\%, deve ser realizada uma adequação do volume do anticoagulante, para que o excesso deste não altere os resultados dos ensaios. Nesse sentido, no intuito de alcançar uma maior eficácia analítica, é recomendável a utilização da concentração de $3,2 \%$ de citrato em um volume total de $56 \%$ de preenchimento de sangue no tubo de coleta. Essas medidas têm resultados de CIN confiáveis ${ }^{28}$.

b) Coleta: a coleta a vácuo é a mais indicada para atender ao objetivo de que o sangue interaja diretamente com o anticoagulante citrato de sódio, presente no tubo de coleta. Se for inevitável a coleta por meio de seringa, esta deve possuir capacidade volumétrica inferior a $10 \mathrm{~mL}$. No ato de dispensar o sangue no tubo, é importante um cuidado especial para evitar hemólise e também para colocar o volume correto ${ }^{11-13}$.

c) Armazenamento amostral: o intervalo entre a punção biológica e a efetiva análise é dependente do exame a ser efetuado e da temperatura de acondicionamento do material biológico. É adequado o processamento das amostras de forma dinâmica e ágili1-13.

A garantia da qualidade mediante a validação analítica dos processos é de fundamental importância. A manutenção da perfeita funcionalidade das centrífugas de forma periódica é essencial para a qualidade da obtenção de matriz plasmática pobre em plaquetas para a realização dos ensaios de coagulação. A recomendação inicial é de que o período entre a coleta e a centrifugação não extrapole 60 minutos. Deve-se selecionar 20 amostras da rotina e mensurar a quantidade plaquetária antes e depois 
da centrifugação, para validar o processo. Após a centrifugação, sem exceção, todas as amostras deverão ter menos de 10.000 plaquetas por $\mathrm{mm}^{311,13}$.

Na rotina de coagulação, é indispensável assegurar a qualidade analítica, trabalhando-se sempre com dois níveis de controle e avaliando as mensurações efetuadas em períodos máximos de 8 horas de rotina $^{29,30}$. Alguns critérios de estabilidade amostral são essenciais: deve-se usar plasma congelado a $-70^{\circ} \mathrm{C}$, no qual o período de estabilidade é maior. No caso de o laboratório possuir a capacidade de congelar a amostra somente a $-20^{\circ} \mathrm{C}$, é de suma importância restringir sua amostragem a 14 dias, no máximo ${ }^{31,32}$. Um considerável grau de padronização é evidente na determinação do CIN para o TP, mas não existem padrões para todos os testes. O ensaio de proficiência é a melhor forma de verificar a exatidão analítica do processo. No entanto, quando ocorre a introdução de um equipamento novo, pode-se compará-lo com o sistema em uso, verificando possíveis inexatidões nas correlações. É importante padronizar o lote dos reagentes para todo o processo, sendo que se houver modificação de lote, as análises anteriores devem ser rejeitadas ${ }^{32}$.

A respeito dos reagentes utilizados no ensaio de coagulação, os estudos ${ }^{17,32-37}$ relatam que, quando há troca de reagente ou de lote do reagente e manutenções nos equipamentos que possam refletir em vulnerabilidades nas ações de validação periódica, são necessários alguns procedimentos específicos, como refazer a curva de calibração, determinar o $\mathrm{CIN}$ e verificar o valor de ISI no laboratório. O TP é o padrão para a determinação do CIN. Os lotes dos reagentes possuem um ISI direcionado e específico, que pode variar de acordo com o equipamento utilizado. Quando há necessidade de introdução de um novo lote ou de troca deste, o laboratório tem de confeccionar uma curva de calibração do sistema e analisar o ISI. Essa atitude atribuirá confiabilidade e rastreabilidade à determinação do $\mathrm{CIN}^{17,32-37}$. Para efetuar esse ensaio, é necessária a utilização de tromboplastina composta de fosfolipídios e fator tissular. O advento do CIN possibilitou reduzir discrepâncias importantes na mensuração do TP, pois leva em consideração a capacidade do reagente de ocasionar a coagulação, favorecendo a comparação dos resultados independentemente da troca de reagentes, lotes ou equipamentos. Para a determinação do CIN, é indispensável o ISI regulamentado pelo fabricante do reagente. O ISI é definido como o índice que relaciona o lote proveniente da tromboplastina do fornecedor com a tromboplastina referenciada internacionalmente. O fornecimento do ISI calculado de forma determinada para o equipamento em uso no laboratório é ideal quando comparado com o ISI geral ${ }^{16,32-37}$.

Adicionalmente, é digna de nota a importância da verificação do ISI no laboratório. Para tanto, utiliza-se três plasmas calibradores com CIN conhecido entre 1,5 e 4,5. Esse teste deve ser realizado em duplicata por três dias. O CIN deve ser estipulado para cada teste e comparado com o CIN esperado (informado pelo calibrador). O resultado satisfatório é definido pelos valores dos CINs contidos em um intervalo de aproximadamente $15,0 \%$ em relação ao CIN esperado. Se o resultado esperado não for alcançado, indica-se elaborar uma nova curva de calibração ou entrar em contato com o fornecedor do reagente para o esclarecimento do ISI específico para o equipamento em questão $0^{15,32-37}$.

\section{Fase Analítica Aplicada à Determinação do TP}

A frequência de fatores que afetam o processo analítico, como amostras contaminadas $(43,0 \%)$, interferência medicamentosa $(29,0 \%)$ e variações laboratoriais $(29,0 \%)$, foram evidenciadas por distintas pesquisas ${ }^{18,23-27}$.

Nos ensaios laboratoriais de coagulação, a realização de testes laboratoriais tem relevância para excluir eventuais interferentes ou possibilitar a identificação da provável fonte de comprometimento dos resultados ${ }^{15}$. Quando o paciente apresenta um resultado alterado, deve-se instantaneamente verificar se está sob efeito de terapêutica anticoagulante, o que justificaria a alteração. Uma alternativa, caso ocorra o contrário, é a realização da adição, na proporção de 1:1, de um pool oriundo de plasma normal com o plasma do paciente. Se este ensaio apresentar resultado normal, é indicativo da presença de inibidores. Se, porventura, não regularizar, conclui-se provavelmente tratar-se de uma coagulopatia ${ }^{15,16,34}$.

A etapa analítica corresponde à fase do processo laboratorial que pode ser regulada pelo controle interno de qualidade $(\mathrm{CIQ})$ e pela avaliação externa da qualidade (AEQ) ou pelos ensaios de proficiência. Para assegurar a qualidade do serviço prestado e, consequentemente, do processo de realização do ensaio, utilizam-se ferramentas como CIQ e AEQ ${ }^{38,39}$. Controles internos com propriedades semelhantes às amostras biológicas são preparados e utilizados na rotina em conjunto com essas ferramentas, com o intuito de avaliar e monitorar a reprodutibilidade e a estabilidade do sistema analítico durante todo o seu fluxo de atuação ${ }^{40}$.

Plebani ${ }^{40}$ ressalta que a dificuldade de estabelecer os erros que ocorrem na fase analítica se dá de acordo com a dificuldade de identificá-los, tendo em vista que cerca de $75,0 \%$ desses erros refletem em resultados na faixa de referência e 12,5\% geram 
valores inteiramente incoerentes, os quais são simultaneamente resolvidos. Assim, assume-se que $12,5 \%$ são os erros que podem influenciar de forma determinante o cuidado e a condição clínica do paciente. Os erros mencionados podem ser reduzidos com a capacitação e a qualificação dos funcionários do laboratório, bem como com a adequação de ações de esclarecimento e correção de falhas de CIQ e AEQ ${ }^{41,42}$.

\section{Fase Pós-analítica Aplicada à Determinação do TP}

A fase pós-analítica inicia-se na área laboratorial e engloba as ações de validação e liberação de laudos. Termina no momento do recebimento do laudo pelo médico, acompanhado de sua interpretação e julgamento de decisão com relação ao resultado registrado no laudo ${ }^{40,41}$.

Equívocos possíveis e visualizados rotineiramente nessa etapa incluem falhas na liberação dos resultados devido a erros de digitação ou transcrição, ou pelo não cumprimento do prazo de entrega. O estabelecimento de ferramentas tecnológicas informacionais direcionadas aos processos de laboratório tem favorecido a redução dos erros de transcrição de resultados. Crucialmente, após o advento dos sistemas de interfaceamento, tornou-se possível a propagação de informações diretamente do equipamento analítico automatizado para o sistema laboratorial de informação ${ }^{42,43}$. Ademais, a etiquetagem com códigos de barras para identificação dos pacientes mostra-se fundamental na busca pelo alcance da melhoria da qualidade e do corte de custos ${ }^{44}$.

Outros procedimentos relacionados à fase pós-analítica são vinculados às informações complementares embutidas no laudo laboratorial, além do resultado. Dizem respeito aos valores de referência e observações que servem de suporte para a interpretação clínica. Os valores de referência de analitos específicos, na grande maioria das vezes, são retirados das instruções do fabricante do kit diagnóstico e podem não ser representativos da população da região em estudo ${ }^{45}$. Da mesma forma, questões relacionadas às informações sobre sensibilidade metodológica, coeficiente de variação, entre outras, deverão ser acrescidas aos laudos, visando representar informações peculiares dos métodos analíticos empregados para a realização dos testes. Erros relacionados a essas informações afetam diretamente a interpretação clínica ${ }^{45,46}$. A garantia da qualidade do laboratório também é dependente do período de tempo de liberação dos resultados, preponderantemente nos exames urgentes ou quando o resultado pode causar um reflexo clínico importante no paciente. Assim, é ímpar a significância de um dinamismo nessa etapa. A lentidão das notificações de valores críticos quase se equivale de forma negativa à liberação de resultados errôneos. Os erros intrínsecos nessa etapa do fluxo laboratorial são de aproximadamente 18,0-47,0\%47,48.

Conforme conclusão de Westgard e Darcy ${ }^{48}$, as repercussões dos resultados laboratoriais representam de $65,0-75,0 \%$ das informações subsidiárias na conduta médica. Portanto, as determinações do TP e CIN de forma adequada e padronizada possuem um impacto considerável no direcionamento médico. Além disso, é de importância vital para os pacientes a comunicação laboratorial de resultados críticos $(\mathrm{CIN}>6,0)$ ou de alertas $(\mathrm{CIN}>4,5-6,0)^{49-51}$. Os possíveis erros nas fases pré-analítica, analítica e pós-analítica ocorrem pela não tomada de medidas para minimizar ou eliminar essas situações. Ademais, em geral, laboratórios de análises clínicas têm uma grande preocupação em garantir a qualidade dos exames e evitar ou minimizar ao máximo os erros, garantindo assim a qualidade e evitando resultados errôneos ${ }^{27,30,33,50}$.

\section{CONSIDERAÇÕES FINAIS}

A dinâmica processual de um laboratório de análises clínicas compreende as fases pré-analítica, analítica e pós-analítica. Essas etapas são influenciadas por uma vasta gama de vulnerabilidades da qualidade, que podem influenciar os resultados dos ensaios laboratoriais e, consequentemente, o tratamento farmacológico do paciente, culminando em um leque de possibilidades de repercussões clínicas. Os analistas clínicos, de forma geral, têm uma importância primordial na avaliação crítica de todas as fases do processo, sendo, portanto, um dos pilares da garantia de resultados condizentes com os aspectos fisiológicos do paciente, fato essencial para que ocorra o efetivo benefício terapêutico dos AVKs.

\section{Conflito de interesse}

Os autores declaram não ter nenhum conflito de interesse.

\section{REFERÊNCIAS}

1. Harenberg J, Hoffmann U, Huhle G, Winkler M, Bayerl C. Cutaneous reactions to anticoagulants: recognition and management. $A m$
J Clin Dermatol. 2001;2(2):6975. PMid:11705306. http://dx.doi. org/10.2165/00128071-20010202000003.
2. Weitz JI, Hirsh J, Samama MM. New antithrombotic drugs. Chest. 2008;133(6 Supl):234S-56S. PMid:18574267. 
3. Kearon C, Kahn SR, Agnelli G, Goldhaber S, Raskob GE, Comerota AJ. Antithrombotic therapy for venous thromboembolic disease. Chest. 2008;133(6 Supl):454S-545S. PMid:18574272.

4. Geerts $W H$, Bergqvist $D$, Pineo GF, Heit JA, Samama CM, Lassen $\mathrm{MR}$, et al. Prevention of venous thromboembolism. Chest. 2008;133(6 Supl):381S-453S. PMid:18574271.

5. Singer DE, Albers GW, Dalen JE, Fang MC, Go AS, Halperin $\mathrm{JL}$, et al. Antithrombotic therapy in atrial fibrillation. Chest. 2008;133(6 Supl):546S-92S. PMid:18574273.

6. Ansell J, Hirsh J, Hylek E, Jacobson A, Crowther M, Palareti G. Pharmacology and management of the vitamin $\mathrm{K}$ antagonists. Chest. 2008;133(6 Supl):160S-98S. PMid:18574265.

7. Comerota A, Ramacciotti E. A comprehensive overview of direct oral anticoagulants for the management of venous thromboembolism. Am J Med Sci. 2016;352(1):92-106. PMid:27432042. http://dx.doi. org/10.1016/j.amjms.2016.03.018.

8. Lima N. Varfarina: uma revisão baseada na evidência das interações alimentares e medicamentosas. Rev Port Clin Geral. 2008;24:475-82.

9. Romano ER, Barbosa MAO, Pinheiro JA Jr. Como iniciar a anticoagulação e sua duração. Rev Soc Cardiol. Estado de São Paulo. 2006;16:256-63.

10. Lima-Oliveira GS, Picheth G, Sumita NM, Scartezini M. The effects of tourniquet application during 1 minute versus subcutaneous tissue transilluminator device in blood sample collection on biochemical parameters. Clin Chem. 2007;S6:123a.

11. Clinical and Laboratory Standards Institute (CLSI). Collection, transport, and processing of blood specimens for testing plasma-based coagulation assays and molecular hemostasis assays: approved guideline. 5. ed. Pennsylvania; 2008.

12. McPherson RA, Pincus MR, Henry JB. Henry's clinical and diagnosis management by laboratory methods. 21. ed. Philadelphia: Saunders Elsevier; 2007.

13. Sociedade Brasileira de Patologia Clínica (SBPC). Recomendações da Sociedade Brasileira de Patologia
Clínica: medicina laboratorial para

coleta de sangue venoso. 2. ed. Barueri: Minha Editora; 2010 [citado 2017 Abr 7]. Disponível em: http:// www.sbpc.org.br/upload/conteudo/ livro_coleta_biologica2013.pdf

14. Carraro P, Plebani M. Errors in a stat laboratory: types and frequencies 10 years later. Clin Chem. 2007;53(7):1338-42. PMid:17525103. http://dx.doi.org/10.1373/ clinchem.2007.088344.

15. Levy JH, Szlam F, Wolberg AS, Winkler A. Clinical use of the activated partial thromboplastin time and prothrombin time for screening: a review of the literature and current guidelines for testing. Clin Lab Med. 2014;34(3):453-77. PMid:25168937. http://dx.doi.org/10.1016/j. cll.2014.06.005.

16. Conway SE, Hwang AY, Ponte CD, Gums JG. Laboratory and Clinical Monitoring of Direct Acting Oral Anticoagulants: What Clinicians Need to Know. Pharmacotherapy. 2017;37(2):236-48. PMid:27983747. http://dx.doi.org/10.1002/phar.1884.

17. Cruz JL, Moss MC, Chen SL, Hansen $\mathrm{KM}$, Amerine LB. Retrospective evaluation of the clinical use of prothrombin complex concentrate for the reversal of anticoagulation with vitamin $\mathrm{K}$ antagonists. Blood Coagul Fibrinolysis. 2015;26(4):378-82. PMid:25688457. http://dx.doi.org/10.1097/ MBC.0000000000000259.

18. Lima-Oliveira G, Volanski W, Lippi G, Picheth G, Guidi GC. Pre-nalytical phase management: a review of the procedures from patient preparation to laboratory analysis. Scand J Clin Lab Invest. 2017;77(3):153-63. PMid:28266238. http://dx.doi.org/10.1 080/00365513.2017.1295317.

19. Sampaio RF, Mancini MC. Estudos de revisão sistemática: um guia para síntese criteriosa da evidência científica. Rev Bras Fisioter. 2007;11(1):83-9. http:// dx.doi.org/10.1590/S141335552007000100013.

20. Kloetzel K, Bertoni AM, Irazoqui MC, Campos VPG, Santos RN. Controle de qualidade em atenção primária à saúde. I. A satisfação do usuário. Cad Saude Publica. 1998;14(3):2638. http://dx.doi.org/10.1590/S0102311X1998000300020.
21. Novaretti MCZ, Bonifacio SL, Medeiros VR, Ruiz AS, Dorlhiac-Llacer PE, Chamone DAF. Dez anos de experiência em controle de qualidade em imuno-hematologia. Rev Bras Hematol Hemoter. 2009;31(3):160-5. http://dx.doi.org/10.1590/S151684842009005000052 .

22. Tenor DP, Laborde CM, Herreros AM, Rodríguez EF. Aproximación matemática para la corrección de la influencia de la hemólisis em pruebas frecuentes del laboratorio clínico. Rev Lab Clin. 2010;3:25-30.

23. Wiwanitkit $\mathrm{V}$. Types and frequency of preanalytical mistakes in the first Thai ISO 9002:1994 certified clinical laboratory, a 6-month monitoring. BMC Clin Pathol. 2001;1(1):5. PMid:11696253. http://dx.doi. org/10.1186/1472-6890-1-5.

24. Berlitz FA. Controle da qualidade no laboratório clínico: alinhando melhoria de processos, confiabilidade e segurança do paciente. $J$ Bras Patol Med Lab. 2010;46(5):353-63. http://dx.doi.org/10.1590/S167624442010000500003.

25. Bonini P, Plebani M, Ceriotti F, Rubboli F. Errors in laboratory medicine. Clin Chem. 2002;48(5):691-8. PMid:11978595.

26. Ferreira CES, Andriolo A. Intervalos de referência no laboratório clínico. $J$ Bras Patol Med Lab. 2008;44(1):116. http://dx.doi.org/10.1590/S167624442008000100004.

27. Loeffen R, Kleinegris MC, Loubele ST, Pluijmen PH, Fens D, van Oerle $R$, et al. Preanalytic variables of thrombin generation: towards a standard procedure and validation of the method. J Thromb Haemost. 2012;10(12):2544-54. PMid:23020632. http://dx.doi.org/10.1111/jth.12012.

28. Potgieter JJ, Pool R, Prinsloo A, Botha EMD, Olorunju S. The impact of collection tube fill volume on international normalized ratio. Med Tech SA. 2010;24:11-6.

29. Clinical and Laboratory Standards Institute (CLSI). Statistical quality control for quantitative measurement procedures: principles and definitions: approved guideline. 3. ed. Pennsylvania; 2006.

30. Clinical and Laboratory Standards Institute (CLSI). Protocol for the evaluation, validation, and implementation of coagulometers: 
approved guideline. Pennsylvania; 2008.

31. Mendes ME, Romano P. Validação de sistema analítico. In: Oliveira CA, Mendes ME. Gestão da fase analítica do laboratório: como assegurar a qualidade na prática. 1. ed. Rio de Janeiro: ControlLab; 2010. vol. 1 [citado 2017 Jan 15]. Disponível em: https://controllab.com/pdf/gestao fase_analitica_vol1.pdf. p. 39-61

32. Clinical and Laboratory Standards Institute (CLSI). Procedures for validation of INR and local calibration of PT/INR systems: approved guideline. Pennsylvania; 2005.

33. Clinical and Laboratory Standards Institute (CLSI). One-stage Prothrombin Time (PT) test and Activated Partial Thromboplastin Time (APTT) Test: approved guideline. 2. ed. Pennsylvania; 2008.

34. Poller L, Ibrahim S, Keown M, Pattison A, Jespersen J. Simplified method for international normalized ratio (INR) derivation based on the prothrombin time/INR line: an international study. Clin Chem. 2010;56(10):160817. PMid:20798354. http://dx.doi. org/10.1373/clinchem.2009.141937.

35. Marlar RA, Gausman JN. Do you report an accurate international normalized ratio? find out using local verification and calibration. Lab Med. 2011;42(3):176-81. http://dx.doi. org/10.1309/LM36LUSEDXEH0GCV.

36. Bonar R, Favaloro EJ, Adcock DM. Quality in coagulation and haemostasis testing. Biochem Med. 2010;20:184-99. http://dx.doi. org/10.11613/BM.2010.023.

37. Berlitz FA, Haussen ML. Seis sigma no laboratório clínico: impacto na gestão de performance analítica dos processos técnicos. $J$ Bras Patol Med Lab. 2005;41(5):301-12. http://dx.doi.org/10.1590/S167624442005000500004.
38. Sciacovelli L, Secchiero S, Zardo L, D'Osualdo A, Plebani M. Risk management in laboratory medicine: quality assurance programs and professional competence. Clin Chem Lab Med. 2007;45(6):75665. PMid:17579529. http://dx.doi. org/10.1515/CCLM.2007.165.

39. Brasil. Ministério da Saúde. Agência Nacional de Vigilância Sanitária. Resolução da Diretoria Colegiada $n^{\circ} 302$, de 13 de out de 2005. Diário Oficial da União. 2005 Out 14 [citado 2017 Jan 15]. Disponível em: http://elegis.anvisa.gov.br/leisref/public/ showAct. php?id=19176\&word=

40. Plebani M. Errors in laboratory medicine and patient safety: the road ahead. Clin Chem Lab Med. 2007;45(6):700-7. PMid:17579520. http://dx.doi.org/10.1515/ CCLM.2007.170.

41. Howanitz PJ, Tetrault GA, Steindel SJ. Clinical laboratory quality control: a costly process now out of control. Clin Chim Acta. 1997;260(2):16374. PMid:9177911. http://dx.doi. org/10.1016/S0009-8981(96)06494-7.

42. Hilt L, Berlin J, Weeks T. Project control for laboratory automation outsourced to consultants: a 10-step process to optimize the effectiveness of custom information technology development. JALA. 2003;8:31-7.

43. Vieira LMF. SBPC/ML: 60 anos. $J$ Bras Patol Med Lab. 2004;40(3):1. http://dx.doi.org/10.1590/S167624442004000300002.

44. Grossi E, Colombo R, Cavuto S, Franzini C. The REALAB project: a new method for the formulation of reference intervals based on current data. Clin Chem. 2005;51(7):123240. PMid:15919879. http://dx.doi. org/10.1373/clinchem.2005.047787.

45. Valenstein PN, Sirota RL. Identification errors in pathology and laboratory medicine. Clin Lab Med. 2004;24(4):979-96, vii. PMid:15555752. http://dx.doi. org/10.1016/j.cll.2004.05.013.

46. Plebani M. Errors in clinical laboratories or errors in laboratory medicine? Clin Chem Lab Med. 2006;44(6):750-9. PMid:16729864. http://dx.doi.org/10.1515/ CCLM.2006.123.

47. Plebani M. Charting the course of medical laboratories in a changing environment. Clin Chim Acta. 2002;319(2):87-100. PMid:11955484. http://dx.doi.org/10.1016/S00098981(02)00028-1.

48. Westgard JO, Darcy T. The truth about quality: medical usefulness and analytical reliability of laboratory tests. Clin Chim Acta. 2004;346(1):311. PMid:15234630. http://dx.doi. org/10.1016/j.cccn.2003.12.034.

49. Ismail AA, Walker PL, Barth JH, Lewandowski KC, Jones R, Burr WA. Wrong biochemistry results: two case reports and observational study in 5310 patients on potentially misleading thyroid-stimulating hormone and gonadotropin immunoassay results. Clin Chem. 2002;48(11):2023-9. PMid:12406989.

50. Bonar R, Favaloro EJ. Explaining and reducing the variation in interlaboratory reported values for International Normalised Ratio. Thromb Res. 2017;150:22-9. PMid:27998809. http://dx.doi. org/10.1016/j.thromres.2016.12.007.

51. Ontario Association of Medical Laboratories (OAML). Guideline for the Laboratory Monitoring of Oral Anticoagulation (Warfarin) CLP014. Ontario; 2008 [citado 2017 Jan 15]. Disponível em: http:// www.oaml.com/PDF/2008/Oral\%20 Anticoagulants $\% 20$ Sept-16-08\%20 -\%20FINAL.pdf

Recebido: Jan 21, 2017 Aceito: Maio 12, 2017 